\title{
Domination of Constrained Application Protocol: A Requirement Approach for Optimization of Internet of Things in Wireless Sensor Networks
}

\author{
P. Madhumitha ${ }^{1 *}$, B. Johnsema' and D. Manivannan ${ }^{2}$ \\ 'School of Computing, Embedded System, SASTRA University, Tirumalaisamudram,Thanjavur-613401, \\ India;madhumithabiw@gmail.com, seema.mtech02@gmail.com \\ ${ }^{2}$ School of Computing, SASTRA University, Tirumalaisamudram,Thanjavur-613401; India; dmv@cse.sastra.edu
}

\begin{abstract}
The protocols related to the Internet of Things (IoT) have proven to undergo changes in the Wireless Sensor Network (WSN), to increase the compatibility of WSN with the industrial and automation applications. In this paper, the programming model, the framework and the security aspects for the Constrained Application Protocol (CoAP) are analyzed with respect to the WSN. CoAP chooses the two-layered approach, to deal with interchanging of messages. The IOT involves implementing the programming in the Representational State Transfer (REST) model paradigm, which is predominantly inherited by the CoAP protocol. The CoAP proxy adapts a backend framework in a modular form. Also, the Datagram Transport Layer Security (DTLS) overcomes unreliable nature in the CoAP due to the User Datagram Protocol (UDP). The CoAP protocol is studied from different angle with respect to the prominent aspects for implementation of IOT in a real world.
\end{abstract}

Keywords: CoAP, IOT, REST, WSN

\section{Introduction}

The tiny embedded devices are linked to the Internet over IP to realize the connection of trillions of smart objects, collectively called as Internet of Things (IoT) or Web of Things. The IoT paradigm is derived and interrelated to Radio Frequency Identification (RFID) systems. The motivations behind choosing the IoT is its importance which can revolutionize in the near-future. As per prediction of the U.S. National Intelligence Council ${ }^{1}$ which lists the IoT in one the five technologies that may impact the U.S. National power by 2025. In addition, the countries like Asia, China and Europe are also involved in promoting the IoTs.

The Internet Engineering Task Force has full-fledgy involved in standardization of the protocols to inte- grate the wireless sensor node such as IRIS mote that possesses the limitation in capacity, power and energy. The COAP is web protocol, similar to HTTP, involved in optimization of the standardization suite. With the development of IPV6 Over Wireless Personal Area Network (6LOWPAN $)^{2}$, the need for integrating the WSN and IoT objects with Internet has increased. The COAP allows the facility to integrate the Representational State Transfer (REST) architecture with the wireless sensor nodes, and consequently connects the WSNs and smart objects with the Web. This feature helps the WSN to reuse the software and lessens the complexities involved during the application development.

In the perspective of the wireless sensor node, the COAP is advantageous over the HTTP in consideration

*Author for correspondence 
to the transport layer. The flow control mechanism of the Transfer Control Protocol (TCP) is not suitable for power constrained networks. It leads to the high overhead for short-lived nodes. This situation is problematic when considering the HTTP as it depends on the control protocol. The COAP is built on the top of the unreliable UDP ${ }^{3}$, thus featuring less overhead and multicast support.

COAP plays the major role in the implementation of message transport mechanism ${ }^{4}$. The communication is established between the client and the node via gateway, which acts as the interface between the SMS and the request to COAP. The gateway forwards the request of the COAP to the node. The SMS is encoded in three categories: 7 bit (160 characters), 8bit (140 characters) and 16 bit (70 characters). Although, the safest solution among these categories is 7-bit encoded SMS i.e. common base 64 encoding for COAP payload.

This paper includes five sections. Section 2 describes the general overview of the COAP and suggests the structured models in the COAP. Section 3 provides the problems to overcome the programming for the IoT. Section 4 entitles the frameworks for COAP that provides the description of the Californium, and the COAP that act as the GUI for the wireless sensor nodes. Section 5 characterizes the analysis of the securing the COAP using the DTLS. Section 6 concludes the importance of the COAP in the future and the compelling needs to optimize the standards of wireless sensor node.

\section{COAP (Constrained Application Protocol)}

The Constrained RESTful Environments (CORE) work aims the REST architecture for 8-bit microcontrollers with limited RAM and ROM. Constrained networks such as 6LOWPAN, support fragmentation and segmentation of IPV6 packets ${ }^{5}$ into small link-layer frames. The design goal of the COAP is to reduce the overhead due to the messages, thus lessening the requirement for the fragmentation of the packets.

The primary aim for designing the COAP is to fulfil the needs of resource-constrained devices, especially dealing with Machine-to-Machine (M2M) ${ }^{6}$ applications. The aim of COAP is to supplement the features of HTTP for automation and $\mathrm{M} 2 \mathrm{M}$ applications. It redesigns or redrafts the HTTP interfaces into a more compact protocol. The main characteristics for COAP are it ensures the need for the $\mathrm{M} 2 \mathrm{M}$, reduces the complexity in parsing and message overhead, supports the URI and resources, includes caching and proxy capabilities and features the message exchange asynchronously.

COAP is a two-layered approach, possessing the features of messaging and request-response models. Broadly, the messaging model deals with asynchronously interchanging the messages via the user datagram protocol. The request/response model deals with request for actions from the client and server of the COAP nodes using the method and response codes. However, the COAP is a single web protocol embedding the features of both the messaging and request/response to the COAP header in the messages. The generalized abstract structure for the WSN model along with two layered approach of the COAP and HTTP is shown in Figure 1.

\subsection{Messaging Model}

COAP messaging model exchanges message over the user datagram oriented transport such as UDP between the client and the server. Each COAP uses a short-fixed length binary reader supplemented with the binary options and the payload. The messages contain a message ID for detecting optional reliability. The reliability is achieved by marking a message as Confirmable (CON). The retransmission of the confirmable message is achieved using a default timeout until the client receives an Acknowledgment message (ACK) with the same message ID from the server. In the Figure 2, the reliable message transmission is depicted along with $\mathrm{CON}$ and ACK. If the ACK is not received within the default timeout, the RST message is sent from the client to the server.

Similarly, there is no need for ACK message in nonreliable transmission messages, instead once the default timeout is expired, the RST message is sent from the client to the server. In Figure 3, the unreliable message transmission is depicted along with NON and RST message.

Since the COAP uses the UDP, it supports the multicast messaging in the network. So, the security is main consideration in the transmission of the packets across the network. The binding of DTLS along with COAP provides the security to the protocol. The usage of IPSec is still in developing stage to provide full-enforcement in the protocol suite.

The message can also be transmitted in unreliable manner using a Non-confirmable message (NON). However, the server does not send the acknowledgment message to the client but possesses the message ID along 


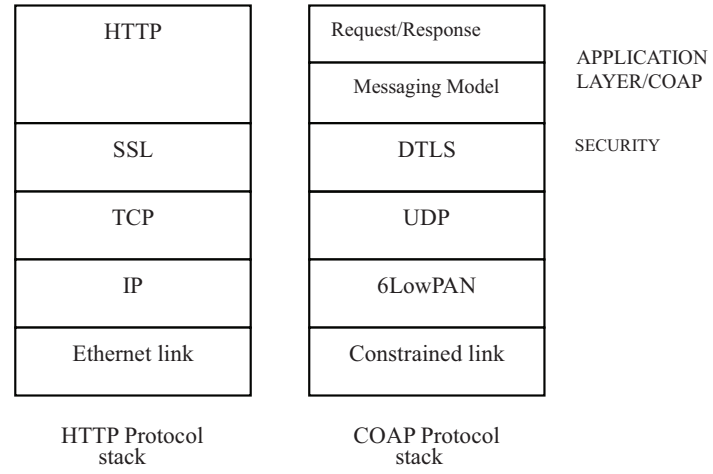

Figure 1. Abstract architecture of COAP for wireless sensor node compared with traditional HTTP.

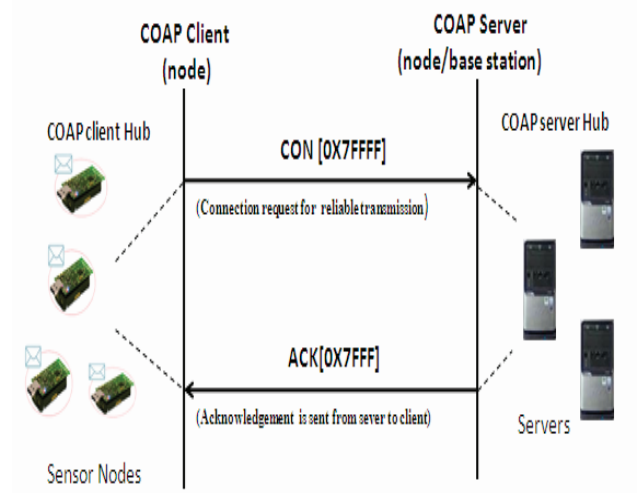

Figure 2. Reliable message transmission in messaging model.

with it for duplicate detection. When the client is not able to process a Non-confirmable message, it may send the Reset message (RST).

\subsection{Request-Response Model}

The semantics of COAP request and response are present in COAP messages, which include either a Method code or a Response code, respectively. A token is used to compare the request and response from the messages. A request is taken in the CON or NON. When the response to the request is taken along with the $\mathrm{ACK}$, then the response is called a piggy-backed response. The COAP makes use of GET, PUT, POST and DELETE methods. In the multicast messages, the server receives more than the requests, the decision is made to which requests, and the server has to respond. If the case arises where server is not able to make the decision about the response, then the request is put in hold and the time duration during which the request is in hold is called leisure ${ }^{6}$.

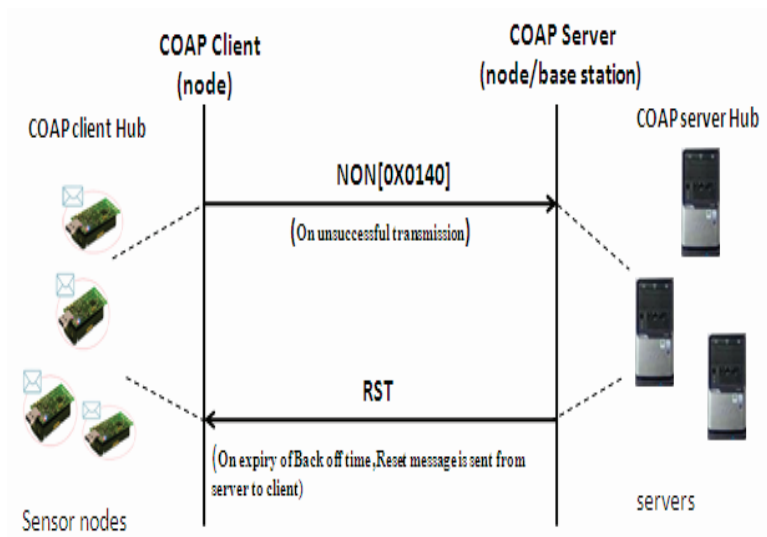

Figure 3. Unreliable message transmission in messaging model.

\section{Programming for IOT}

The growth of IoT has invoked to develop the programming language for compatible interactivities among the devices. The issues in the programming for IoT are the ideas with respect to low-power networking, a resource-constrained operating system and a distributed algorithm. Usually, the application code is traditionally written in the topmost layer of the Operating System (OS) such that the code is statically linked to the OS. This way of implementation is efficient but more error prone as the developers need to have an idea of the OS. Another alternative method is to implement the virtual machine in the device. The first virtual machine for the sensor network is the Mate ${ }^{7}$. In the paper ${ }^{8}$, the Darjeeling virtual machine is written in the Java language for deploying the large heterogeneous WSN. Both the VM minimizes overhead and provides the dynamic features. However, the application architecture for the IoT is well-suited COAP that is light-weighted UDP on basis of RESTFUL interaction to the low-constrained devices.

In the network embedded system, the scripting language organizes the well-defined batch operations in the WSN. The scripts ensure the self-contained network and provide the focused the functionalities inthe networking such that the interactivity among the IoT devices are enhanced in an easier manner. The scripts are usually compiled before transferring to the device using the sc Python. The applications in the WSNs are realized using the scripts executed on the server in the cloud. The attempt is made to use the existing widely used language in the Web, specifically Java script. In addition, the 
enhancement of an Application Programming Interface (API) for RESTful interaction with the mote based devices. The IoT applications are implemented using the Actinuim (Ac) apps in the RESTful model paradigm. The resources of the IoT are interacted using the GET, the POST and the PUT methods. The complex apps is broken into modular scripts that communicates through the RESTful interfaces. This concept features the reusability of the modules in the other apps. Thus, mash-up concept is made efficient in the homogenous and heterogeneous IoT devices. The scripts are contained in a run-time container that provides the resources and an application object API. This container serves as an app server for providing the dynamic installation, the updates and the removal of the scripts.

\section{Frameworks for COAP}

The interoperability in the application layer requires thin server architecture for the IoTs. The real-time embedded system is integrated with the Web for adaptation of computer to react towards the physical phenomenon in the real world. This provides the opportunities for the existing services and tools to deal with the real-time problems. The intuitive APIs are used for public services to interconnect seamlessly.

Californium $^{9}$ and Erbium are the back-end frameworks which act as the COAP proxy. The Californium framework includes adverse layer to test packet losses at different scenarios, Base layer that includes UDP for transport mechanism and other new layers can be easily added as per requirements such as in case of security. The hierarchy technique is implemented in linking resources to each other. CORE link format is used to add the resource in the hierarchy. One of the frameworks for the COAP is the Californium written in language Java, which supports the back-end client, the server and the proxies. The design of the Californium is modular in nature. It deploys the COAP resources in a back-end. In addition, it acts as bridge between the HTTP and the COAP using a default Java Http server. The COAP is accessed by the respective request object that is similar to XML HTTP object.

In addition, there are two ways to access the resources using a forward-proxy namely CoAP-HTTP proxying and HTTP-CoAP proxying ${ }^{6}$. Firstly, considering the CoAPHTTP proxying, the CoAP clients access the resources using the proxy-uri with http or https URI on the HTTP servers. Secondly, considering the HTTP-CoAP proxying, the HTTP clients access the resources using the coap or coaps URI on CoAP servers.

\section{Securing COAP with DTLS}

The issues of security are primary concern in the communication system. In COAP, the security is one of primary requirement for authorization and authentication in the protocol. It is either provided by communication security $^{10}$ or object security. The communication security is analogy for IPSec and DTLS ${ }^{3}$. The object security is synonyms for providing security along with payload. The intermediaries require the communications security such that it is assumed that the intermediaries are included in the trust relationships. COAP doesn't allow the packets to reach the servers from the client until the security check is provided in the intermediaries of the network. Comparing the Transfer Control Protocol (TCP) ${ }^{11}$ over the UDP, the HTTP is provided with security using the transfer layer security and the COAP is secured using the DTLS. Thus, the DTLS partially overcomes unreliable nature of the UDP.

\section{Conclusion}

CoAP is realized to be more adaptable and reliable in comparison to the HTTP for constrained WSN nodes. The protocol is optimized with respect to the framework and security for implementation in the IOT applications. In this work, the overall analysis of web protocol, the CoAP is realized with respect to the IOT. In addition, the COAP is more effective in comparison to the HTTP as it reduces the header overhead and supports the multicast messages. The features of the request to response model are dominantly inherited in the abstract two-layered model of COAP. The security is provided via the DTLS layer to support the unreliable UDP in the abstract architecture. Also, the research activities in the COAP optimize the imagination of future in the IoT. In the future work, we are involved in the implementation of the COAP protocol in the IRIS mote.

\section{References}

1. Iera A, Floerkemeier C, Mitsugi J, Morabito G. Internet of things, IEEE Wireless Communications, 2010 Dec ; 17(6): 8-9.

2. Raza S, Trabalza D, Voigt T. 6LoWPAN compressed DTLS for CoAP. 2012 8th IEEE International Conference 
on Distributed Computing in Sensor Systems; 2012 May 16-18; China: Conference Center of Zhejiang University Hangzhou: Distributed Computing in Sensor Systems (DCOSS); 2012. p. 287-289.

3. Bormann C, Castellani AP, Shelby Z. CoAP: An application protocol for billions of tiny internet nodes. IEEE Computer Society. 2012; 16(2):62-67.

4. Gligoric N, Dimcic T, Drajic D, Krco S, Dejanovic I, Chu N, Obradovic A. CoAP over SMS: performance evaluation for machine to machine communication. 20th Telecommunications forum TELFOR; Serbia: Belgrade; 2012 Nov 20-22, 2012 Valencia: Mobile Adhoc and Sensor Systems (MASS); 2011. p. 855-860.

5. Mattias Kovatsch, Simon Duquennoy and Adam Dunkels. A Low-Power COAP for Contiki, 2011 Eighth IEEE International Conference on Mobile Ad-Hoc and Sensor Systems.

6. ShelbyZ, Hartke K, Bormann C. Draft-IETF-core-COAP-18, 2013; 2-25.

7. Levis P, Culler D. Mate: a tiny virtual machine for sensor networks. Proceedings of the 10th annual conference on
Architectural Support for Programming Languages and Operating Systems; 2008 Nov 5-7; USA: Raleigh, North Carolina. Dec 2002; New York, NY, USA. p. 85-95.

8. Brouwers N, Langendoen K, Corke P. Darjeeling, aFeaturerich VM for the resource poor. Proceedings of the 7th ACM Conference on Embedded Networked Sensor Systems. 2009 November 4-6; USA: Berkeley, CA 2009; New York, NY, USA. p. 169-182.

9. Kovatsch M, Mayer S, Ostermaier B. Moving application logic from the firmware to the cloud: towards the thin server architecture for the internet of things. Proc. IMIS, Palermo. 2012; Italy 2012; Palermo: Innovative Mobile and Internet Services in Ubiquitous Computing (IMIS). p. 751-756.

10. Kuladinithi K, Bergmann O, Pötsch T, Becker M, Görg C. Implementation of CoAP and its application in transport logistics. In Proc.IP+SN.2011; USA: Chicago, IL 2011; Chicago, IL, USA. p. 1-7.

11. Garcia-Morchon O, Keoh S, Kumar S, Hummen R, Struik R.Internet-DraftSecurityConsiderationsfortheIoT.Mar2013; 1-45. 